\title{
Força muscular respiratória e sua relação com a idade em idosos de sessenta a noventa anos
}

\author{
Rodrigo Polaquini Simões", Viviane Castello", Marco Antonio Auad"*, \\ Jadiane Dionísio****, Marisa Mazzonetto
}

\section{Resumo}

Os objetivos deste estudo foram comparar a força muscular respiratória (FMR) entre idosos aparentemente saudáveis e verificar se há relação entre a FMR e a faixa etária nesta população. Foram estudados sessenta idosos com idade entre sessenta e noventa anos, sendo separados em dois grupos, com trinta homens e trinta mulheres. Cada grupo foi dividido em três subgrupos de acordo com a faixa etária: 60 a 69 anos, 70 a 79 anos e 80 a 90 anos. Todos foram submetidos a um teste de manovacuometria para determinação das pressões inspiratória máxima (PImáx) e expiratória máxima (PEmáx). Os valores das pressões respiratórias máximas (PRM) foram comparados entre os subgrupos em cada grupo, e análise de correlação foi aplicada entre a idade e os valores das PRM. O nível de significância adotado foi $p<0,05$. Foram verificados valores significativamente menores de PImáx e PEmáx do subgrupo de 70-79 anos em relação ao de 80-90 anos; do subgrupo de 60-69 anos em relação ao de 70-79 anos, tal como em relação ao de 80-90 anos, em ambos os grupos. Correlações significativas e negativas foram verificadas em ambos os grupos tanto entre a idade e a Plmáx (homens: $R=-0,83$; muIheres: $R=-0,86$ ) como entre a idade e a PEmáx (homens: $R=-0,88$; mulheres: $R=$ $-0,86)$. Conclui-se que a FMR se reduz com o avançar da idade em idosos de sessenta a noventa anos e que há forte relação entre a idade e a FMR nesta população.

Palavras-chave: Idoso. Sistema respiratório. Músculos respiratórios. Força muscular.

* Doutorando em Fisioterapia no Programa de Pós-Graduação em Fisioterapia da Universidade Federal de São Carlos. Endereço para correspondência: Laboratório de Fisioterapia Cardiorrespiratória, Departamento de Fisioterapia, Universidade Federal de São Carlos, Rodovia Washington Luís, km 235, bairro Monjolinho, CEP: 13565-905, São Carlos - SP. E-mail: rpssimoes@yahoo.com.br.

** Mestranda em Fisioterapia no Programa de Pós-Graduação em Fisioterapia da Universidade Federal de São Carlos.

**** Mestrando em Biotecnologia no Programa de Pós-Graduação em Biotecnologia da Universidade Federal de São Carlos.

**** Mestranda em Fisioterapia no Programa de Pós-Graduação em Fisioterapia da Universidade Federal de São Carlos.

***** Mestre em Cirurgia pela Universidade Estadual de Campinas. Professora de Fisioterapia Cardiorrespiratória da Universidade Camilo Castelo Branco, campus VIII.

$\rightarrow$ Recebido em maio de 2009 - Avaliado em março de 2010.

$\rightarrow$ doi: $10.5335 /$ rbceh.2010.006 


\section{Introdução}

No Brasil e no mundo, o número de pessoas com idade acima de sessenta anos vem crescendo significativamente, tanto que no ano de 2006 a população idosa no país passou a representar 19 milhões, ou seja, mais de $10 \%$ da população total. A expectativa do número de idosos ao final dos próximos vinte anos é de mais de trinta milhões, representando cerca de $13 \%$ da população total do país. (IBGE, 2009).

Com o processo de senescência, mudanças no sistema respiratório dos idosos são verificadas, tal como alterações na quantidade e composição dos componentes dos tecidos conjuntivos do pulmão e da caixa torácica, como da elastina, colágeno e proteoglicanos. (KIM; SAPIENZA, 2005; CHAUNCHAIYAKUL et al., 2004; JANSSENS; PACHE; NICOD, 1999). Outro fator de grande importância é a perda de massa muscular, denominada de "sarcopenia", que acomete não só o sistema muscular periférico, mas também os músculos que atuam na respiração. (ENRIGHT et al., 1994).

A fraqueza dos músculos respiratórios pode resultar em dificuldades dos pulmões em captar o oxigênio do ar atmosférico em razão da deficiência na mecânica respiratória. (POWERS; CRISWELL, 1996). Esse fato se agrava em situações de esforço físico, gerando redução na tolerância ao exercício. (WATSFORD; MURPHY; PINE, 2007). A fraqueza desses músculos em indivíduos idosos pode dificultar até mesmo atividades de vida diária comuns, as quais exigem pequenos esforços físicos (WATSFORD; MURPHY, 2008). No entanto, isso pode se tornar mais grave nos casos em que estes indivíduos são acometidos por patologias como pneumonia e insuficiência ventricular esquerda, pois sobrecarregam ainda mais os músculos respiratórios, o que pode resultar em insuficiência respiratória. (SHARMA; GOODWIN, 2006; HARIK-KHAN; WISE; FOZARD, 1998). Nesse sentido, a avaliação da força muscular respiratória (FMR) é de grande importância nesta população, já que pode auxiliar na avaliação da integridade do sistema respiratório. (McCONNELL; COPESTAKE, 1999; SUMMERHILL, 2007; VINCKEN; GHEZZO; COSIO, 1987).

AFMR pode ser mensurada por meio das pressões sub e supra-atmosféricas, geradas pelos músculos inspiratórios e expiratórios, respectivamente. Essas pressões, avaliadas na boca, refletem a pressão que é gerada nos alvéolos pela ação dos músculos respiratórios. (CAMELO; TERRA-FILHO; MANÇO, 1985; MIZUNO, 1991; HARIK-KHAN; WISE; FOZARD, 1998; STEIER et al., 2007). A maior pressão que pode ser gerada durante a inspiração forçada contra uma via aérea ocluída é denominada de "pressão inspiratória máxima" (PImáx), e a maior pressão que pode ser desenvolvida durante um vigoroso esforço expiratório contra uma via aérea ocluída, de "pressão expiratória máxima” (PEmáx). (NEDER et al., 1999). A análise da FMR por meio das pressões respiratórias máximas (PRM) tem sido muito utilizada na prática clínica em razão do seu importante papel diagnóstico 
e prognóstico em disfunções que causem a fraqueza dos músculos respiratórios, sendo considerado um método simples e prático, não invasivo, de baixo custo e de grande representatividade e confiabilidade. (MAN et al., 2003; HAMNEGÅRD; GREEN, 1994).

Muitos estudos já comprovaram que há diferença na força dos músculos respiratórios entre jovens e idosos, tal como relação negativa da idade com a FMR (ENRIGHT et al., 1994; VINCKEN; GHEZZO; COSIO, 1987; NEDER et al., 1999). No entanto, não são de nosso conhecimento estudos que tenham verificado se há variação significativa da força desses músculos em uma amostra composta exclusivamente por indivíduos idosos saudáveis. Portanto, os objetivos deste estudo foram comparar a FMR entre idosos aparentemente saudáveis de sessenta a noventa anos de idade e verificar se há relação entre a FMR e a faixa etária nesta população.

\section{Materiais e métodos}

\section{Casuística}

Foram estudados sessenta idosos com idade entre sessenta e noventa anos, separados em dois grupos, com trinta homens $(\mathrm{H})$ e trinta mulheres $(\mathrm{M})$ cada. Cada um desses grupos foi dividido em três subgrupos com dez idosos, de acordo com a faixa etária: 60 a 69 anos, 70 a 79 anos e 80 a 90 anos. Todos os procedimentos foram explicados aos participantes, bem como descritos detalhadamente no termo de consentimento assinado previamente por eles. O estudo foi analisado e aprovado pelo Comitê de
Ética em Pesquisas com Seres Humanos da universidade e seguiu a resolução 196/96 do Conselho Nacional de Saúde.

\section{Critérios de inclusão}

Foram selecionados idosos classificados como eutróficos ou sobrepeso (índice de massa corpórea - IMC de 18,5 a 29,9 $\mathrm{kg} / \mathrm{m}^{2}$ ) segundo a Organização Mundial de Saúde (2000), que não praticassem nenhum tipo de atividade física regularmente, não fumantes, na ausência de doenças respiratórias (agudas ou crônicas) ou neuromusculares, como também de retrações cutâneas ou desvios estruturais acentuados na coluna que pudessem interferir na dinâmica respiratória.

\section{Protocolo experimental}

Inicialmente, os voluntários passaram por uma avaliação constituída da história clínica prévia, exames físicos e anamnese, sendo realizado o registro dos dados pessoais, das doenças e cirurgias prévias, análise visual para identificação de possíveis alterações na região torácica e abdominal e desvios acentuados da coluna, além de ausculta pulmonar, cardíaca, medida da temperatura corporal, frequência cardíaca e pressão arterial. Posteriormente, as características antropométricas foram avaliadas, sendo a estatura determinada por um estadiômetro e a massa corporal, por uma balança previamente calibrada (Welmy, São Paulo, SP, Brasil). Ambas as medidas foram realizadas com os sujeitos descalços e utilizando roupas leves. O IMC foi calculado para cada voluntário por meio da razão do peso corporal pela altura elevada ao quadrado (peso/altura ${ }^{2}-\mathrm{kg} / \mathrm{m}^{2}$ ). 
Para avaliação das PRM foi utilizado um manovacuômetro do tipo aneroide (Gerar MV300, São Paulo, SP, Brasil) com intervalo operacional de \pm 300 $\mathrm{cmH}_{2} \mathrm{O}$. Um tubo plástico foi conectado ao aparelho e em sua extremidade distal foi adaptado um dispositivo plástico com um pequeno orifício, de aproximadamente $2 \mathrm{~mm}$ de diâmetro (CHETTA et al., 2001), que permitia pequeno vazamento de ar, evitando a elevação da pressão da cavidade oral gerada pela contração da musculatura facial (BLACK; HYATT, 1969). Para cada indivíduo foi utilizado um bocal de borracha com $32 \mathrm{~mm}$ de diâmetro, que era conectado ao dispositivo plástico citado.

Previamente à realização das medidas, o voluntário era orientado a permanecer na posição sentado, sendo realizada a familiarização com o equipamento. Após a colocação do clipe nasal e o posicionamento adequado do bocal na cavidade oral (para não permitir escape de ar) o voluntário realizava, primeiramente, a manobra de inspiração forçada (PImáx) a partir do volume residual, repetindo-a pelo menos três vezes, com intervalo de um minuto entre cada repetição. Posteriormente, a manobra de expiração forçada (PEmáx) era realizada a partir da capacidade pulmonar total, utilizando-se a mesma metodologia aplicada durante a manobra inspiratória. Todas as medidas foram realizadas por um único avaliador sob comando verbal homogêneo. O esforço respiratório deveria ser sustentado em seu máximo por aproximadamente um segundo (NEDER et al., 1999); o maior valor obtido de três repetições corretamente realizadas em cada manobra (com diferença de $10 \%$ ou menos entre os valores) foi o valor considerado da PImáx e da PEmáx. (NEDER et al., 1999).

\section{Análise estatística}

A distribuição dos dados foi verificada por meio do teste de Shapiro-Wilk e, após constatada a normalidade, esses foram expressos em média e desviopadrão. Para comparar as características antropométricas entre os grupos (homem versus mulheres) foi utilizado o teste $t$ de Student para amostras independentes. Para comparar as características antropométricas, tais como as pressões respiratórias máximas (PImáx e PEmáx), entre os subgrupos em cada grupo foi utilizada a análise de variância (Anova) com pós-teste de Tukey Kramer.

O coeficiente de correlação linear de Pearson foi utilizado para verificar o nível de correlação entre a idade e os valores das pressões respiratórias máximas, tanto para o grupo dos homens como para o das mulheres. A probabilidade de ocorrência de erro do tipo I foi estabelecida em 5\% para todos os testes. Os dados foram analisados pelos programas Statistica for Windows (StatSoft Inc, 2000) e Statistical Package for the Social Sciences ${ }^{\mathrm{TM}}$ (SPSS - IBM, versão 10.0.1, 1999).

\section{Resultados}

Quando comparadas a idade e as características antropométricas entre o grupo de homens e o das mulheres, tal como entre os subgrupos em cada grupo, verificou-se diferença significativa da 
idade entre os subgrupos (tanto para os homens como para as mulheres). Em relação às variáveis antropométricas massa corporal, estatura e IMC - não foi observada diferença significativa tanto intergrupo como intragrupo (Tabela 1).

Em relação às PRM, foram verificados valores significativamente menores da PImáx e da PEmáx do subgrupo de 70 a 79 anos em relação ao de 80 a 90 anos; do subgrupo de 60 a 69 anos em relação ao de 70 a 79 anos, tal como em relação ao de 80-90 anos, tanto no grupo dos homens como no grupo das mulheres (Figura 1, A e B). Foram verificadas correlações significativas e negativas tanto para os homens como para as mulheres entre a idade e a PImáx (homens: $\mathrm{R}=$ $-0,83$; mulheres: $R=-0,86$ ) e a idade e a PEmáx (homens: $\mathrm{R}=-0,88$; mulheres: $R=-0,86)$, como observado na Figura 2 (A e B, respectivamente).

Tabela 1 - Idade e características antropométricas dos voluntários separados em grupos de acordo com o gênero e em subgrupos de acordo com a faixa etária.

\begin{tabular}{cccccc}
\hline Sexo & Faixa etária & Idade (anos) & Massa $(\mathrm{kg})$ & Estatura $(\mathrm{m})$ & $\mathrm{IMC}\left(\mathrm{kg} / \mathrm{m}^{2}\right)$ \\
\hline \multirow{3}{*}{ Homens } & De 60 a 69 anos & $64,8 \pm 3,6$ & $63,61 \pm 2,86$ & $1,63 \pm 0,04$ & $23,81 \pm 1,58$ \\
& De 70 a 79 anos & $74,9 \pm 3,1^{*}$ & $64,20 \pm 5,07$ & $1,64 \pm 0,05$ & $23,75 \pm 1,52$ \\
& De 80 a 90 anos & $84,8 \pm 2,3^{*} \#$ & $62,36 \pm 2,83$ & $1,60 \pm 0,03$ & $24,18 \pm 1,04$ \\
\multirow{3}{*}{ Mulheres } & De 60 a 69 anos & $65,1 \pm 2,6$ & $66,22 \pm 3,14$ & $1,61 \pm 0,03$ & $25,52 \pm 1,27$ \\
& De 70 a 79 anos & $74,8 \pm 2,5^{*}$ & $62,87 \pm 3,74$ & $1,59 \pm 0,06$ & $24,40 \pm 1,82$ \\
& De 80 a 90 anos & $85,1 \pm 3,4^{*}$ & $63,09 \pm 3,47$ & $1,58 \pm 0,02$ & $24,69 \pm 0,97$ \\
\hline
\end{tabular}

Dados expressos em media e desvio-padrão. IMC = índice de massa corpórea. "Diferença significativa em relação ao subgrupo de 60 a 69 anos; "Diferença significativa em relação ao subgrupo de 70 a 79 anos.
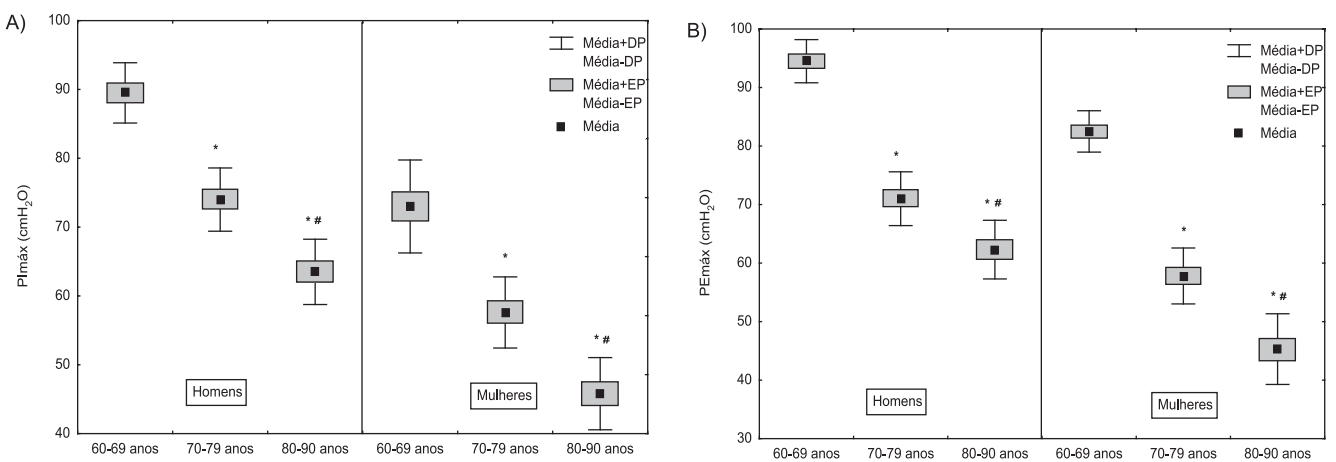

Figura 1 - Em A, comparação dos valores médios da PImáx (pressão inspiratória máxima) entre os subgrupos em cada grupo (homens à esquerda e mulheres à direita). Em B, comparação dos valores da PEmáx (pressão expiratória máxima) entre os subgrupos em cada grupo (homens à esquerda e mulheres à direita). $\mathrm{DP}=$ Desvio- padrão; $\mathrm{EP}=$ Erro padrão. *Diferença significativa em relação ao subgrupo de 60 a 69 anos. \#Diferença significativa em relação ao subgrupo de 70 a 79 anos. 

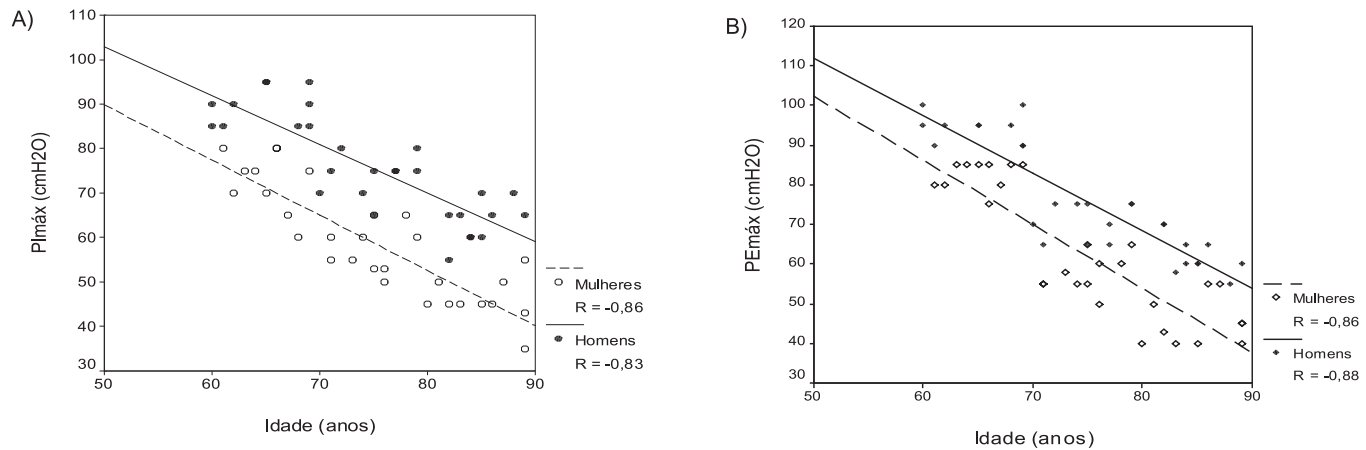

Figura 2 - Em A, correlação negativa e significativa da PImáx (pressão inspiratória máxima) e em B, da PEmáx (pressão expiratória máxima) com a idade tanto em homens (círculos em A e losangos em B, cheios) como em mulheres (círculos em A e losangos em B, vazios).

\section{Discussão}

A FMR tem sido apresentada como um importante parâmetro para avaliação da integridade do sistema respiratório, principalmente em idosos, os quais sofrem modificações importantes nesse sistema com o processo de senescência, podendo causar limitações em suas atividades de vida diária. Este manuscrito traz novas contribuições em relação ao estudo da FMR exclusivamente de idosos saudáveis, com peso dentro da faixa de normalidade e que não tiveram história de tabagismo, considerando o fato de que estudos prévios demonstraram influências desses fatores sobre a FMR.

Os principais achados deste estudo revelaram que a idade influencia na FMR de idosos saudáveis com faixa etária entre sessenta e noventa anos, já que observamos que as PRM foram significativamente menores nos indivíduos com maior idade. Em relação às características antropométricas, o fato de não haver diferença tanto intragrupo como intergrupo confirma a homogeneidade da amostra. Por sua vez, em relação à idade verificou-se diferença significativa entre os subgrupos de ambos os grupos, fato já esperado por se separarem os subgrupos por décadas (Tabela 1).

Tanto nos homens como nas mulheres, observou-se diminuição progressiva e significativa da PImáx e da PEmáx com o avançar de cada década a partir dos sessenta até os noventa anos (Figura 1, A e B). Essas reduções nos valores pressóricos são indícios de que perda da força da musculatura inspiratória e expiratória ocorre nos idosos com maior faixa etária, estando diretamente relacionadas ao processo de envelhecimento, fato confirmado pelas fortes correlações negativas encontradas entre a idade $\mathrm{e}$ as PRM (Figura 2, A e B).

Tolep et al. (1995) compararam a força da musculatura diafragmática de jovens de 19 a 28 anos com idosos de 65 a 75 anos. Os autores verificaram redu- 
ção significativa da força do diafragma nos indivíduos idosos e concluíram que o decréscimo da força desta musculatura relacionada com a idade poderia predispor esses idosos à fadiga diafragmática na presença de condições que exigissem maior esforço desta musculatura, como, por exemplo, durante o exercício físico, no qual os músculos respiratórios são mais recrutados, principalmente durante níveis mais elevados de ventilação.

Enright et al. (1994) avaliaram as pressões respiratórias máximas de idosos com idade superior a 65 anos, os quais foram separados em subgrupos de acordo com a faixa etária (65-69, 70-74, 75-79, 80-84 e maiores de 85 anos). Os autores verificaram correlação negativa das PRM com a idade, ou seja, redução nos valores dessas pressões com 0 aumento da faixa etária dos referidos subgrupos.

Nossos resultados corroboram os estudos de Tolep et al. (1995) e de Enright et al. (1994), e nesse sentido supomos que vários fatores podem ter sido responsáveis pela redução da FMR com 0 aumento da idade, como, por exemplo, alterações da atividade e do perfil das enzimas glicolíticas e anaeróbicas, diminuição da densidade capilar, redução da velocidade de encurtamento e geração de tensão do diafragma, substituição parcial do tecido contrátil por não contrátil, alterações na junção neuromuscular, entre outros. (ENRIGHT et al., 1994; BLACK; HYATT, 1969; JANSSENS; PACHE; NICOD, 1999; TOLEP et al., 1995; TOLEP; KELSEN, 1993).

No entanto, acreditamos que os principais fatores que podem influen- ciar fortemente na redução da FMR com o envelhecimento são: diminuição da massa da musculatura respiratória, que acarretaria redução da eficiência e, consequentemente, diminuição da força muscular, assim como alterações estruturais, principalmente na região torácica desses indivíduos.

O processo de perda de massa muscular é denominado de sarcopenia e se desenvolve com a progressão da idade. (DOHERTY, 2003). Com a redução da massa, a musculatura respiratória perde sua eficiência em gerar força, fato que se reflete em menores valores das pressões respiratórias máximas. (TOLEP; KELSEN, 1993). Mizuno et al. (1991) reportaram em seu trabalho que a área de secção transversal dos músculos intercostais internos diminui aproximadamente 7 a $20 \%$ em indivíduos com cinquenta anos de idade em virtude da redução nas fibras do tipo I e, principalmente, do tipo II. Segundo Kim e Sapienza (2005), esse decréscimo da massa muscular relacionado à idade pode prejudicar tanto a função de bomba ventilatória como as funções não ventilatórias do sistema respiratório, como tossir, espirrar, falar, cantar, entre outras.

As mudanças estruturais do tórax do idoso também devem ser consideradas em relação à força dos músculos respiratórios. A articulação sinovial entre 0 esterno e as cartilagens costais desaparece e os elementos ósseos e cartilaginosos se fundem; os muitos condrócitos das cartilagens costais se degeneram e as fibras colágenas se espessam; ainda, aumenta o depósito de cálcio e a cartila- 
gem fica mais rígida. (KIM; SAPIENZA, 2005; CHAUNCHAIYAKUL et al., 2004; JANSSENS; PACHE; NICOD, 1999). Como a mobilidade da caixa torácica na respiração depende da movimentação das articulações, as alterações citadas provocam aumento da complacência pulmonar, ao passo que reduzem as forças de tração da parede torácica. (CHAUNCHAIYAKUL et al., 2004; JANSSENS; PACHE; NICOD, 1999). Além disso, o processo de osteoporose também pode influenciar nas alterações da configuração do tórax, já que pode causar microfraturas parciais ou completas das vértebras, levando ao aumento da cifose dorsal e do diâmetro anteroposterior do tórax. (JANSSENS; PACHE; NICOD, 1999).

Como a mobilidade da caixa torácica na respiração depende da movimentação das articulações, as alterações estruturais citadas provocam redução das forças de tração da parede torácica, desencadeando a diminuição das pressões respiratórias máximas. Além disso, essas modificações estruturais da caixa torácica alteram a curvatura do músculo diafragma, provocando um efeito negativo em sua capacidade de gerar força. (POLKEY; GREEN; MOXHAM, 1995).

\section{Conclusão}

Diante do exposto, conclui-se que os valores das pressões respiratórias máximas (PImáx e PEmáx) se reduzem significativamente com o avançar de cada década em idosos de sessenta a noventa anos e que há forte relação negativa entre a idade e a FMR neste população.
Relationship between respiratory muscle strength and age in elderly sixty to ninety years old

\section{Abstract}

The purpose of this study were to compare the respiratory muscle strength (RMS) among apparently healthy elderly people and verify if there is relationship between the RMS and age in this population. The participants were 60 elderly with age between 60 and 90 years, separated into two groups: men $(n=30)$ and women $(n=30)$. Each group was divided into three subgroups according to age: 60 to 69 years, 70 to 79 years and 80 to 90 years. All were submitted to manovacuometry test for determination of maximal inspiratory pressure (MIP) and maximal expiratory pressure (MEP). The values of maximal respiratory pressures (MRP) were compared among subgroups in each group, moreover, correlation analysis was applied between age and MRP values. The level of significance was $p<0,05$. Were found significantly lower values of MIP and MEP of the subgroup of 70-79 years in relation to $80-90$ years subgroup; of 60-69 years subgroup in relation to $70-79$ years subgroup, as in relation to 80-90 years subgroup, in both groups (men and women). Significant and negative correlations were observed in both groups between age and MIP (males: $R=-0,83$, females: $R=-0,86)$ and between age and MEP (males: $R=-0,88$, females: $R$ $=-0,86)$. In conclusion, the RMS decrease with age in elderly 60 to 90 years and there is strong relationship between age and RMS in this population.

Key words: Elderly. Respiratory system. Respiratory muscles. Muscle strength. 


\section{Referências}

BLACK, L. F.; HYATT, R. E. Maximal respiratory pressures: normal values and relationship to age and sex. Am. Rev. Respir. Dis., v. 99 , n. 5, p. 696-702, 1969.

CAMELO Júnior, J. S.; TERRA-FILHO J.; MANÇO, J. C. Pressões respiratórias máximas em adultos normais. J. Pneumol., v. 181, n. 4, p. 181-184, 1985.

CHAUNCHAIYAKUL, R. et al. The impact of aging and habitual physical activity on static respiratory work at rest and during exercise. Am. J. Physiol. Lung. Cell. Mol. Physiol., v. 287, n. 6, p. 1098-1106, 2004.

CHETTA, A. et al. Whistle mouth pressure as test of expiratory muscle strength. Eur. Respir. J., v. 17, n. 4, p. 688-695, 2001.

DOHERTY, T. J. Invited review: aging and sarcopenia. J. Appl. Physiol., v. 95, n. 4, p. 1717-1727, 2003.

ENRIGHT, P. L. et al. Respiratory muscle strength in the elderly. Correlates and reference values. Cardiovascular Health Study Research Group. Am. J. Respir. Crit. Care Med., v. 149, n. 2, pt. 1, p. 430-438, 1994.

HAMNEGÅR, C. H.; GREEN, M. Portable measurement of maximum mouth pressures. Eur. Respir. J., v. 7, n. 2, p. 398-401, 1994.

HARIK-KHAN, R. I.; WISE, R. A.; FOZARD, J. L. Determinants of maximal inspiratory pressure: The Baltimore longitudinal study of aging. Am. J. Respir. Crit. Care Med., v. 158 , n. 5 , pt. 1 , p. 1459-1464, 1998.

IBGE. Ministério do Planejamento, Orçamento e Gestão, Brasil. Perfil dos idosos responsáveis pelos domicílios. 2002. Disponível em: <www.ibge.gov.br/home/presidencia/ noticias/25072002pidoso.shtm>. Acesso em: 10 mar. 2009.

JANSSENS, J. P.; PACHE, J. C.; NICOD, L. $P$. Physiological changes in respiratory function associated with ageing. Eur. Respir. J., v. 13, n. 1, p. 197-205, 1999.
KIM, J.; SAPIENZA, C. M. Implications of expiratory muscle strength training for rehabilitation of the elderly: tutorial. JRRD, v. 42 , n. 2, p. 211-224, 2005.

MAN, W. D. et al. Cough gastric pressure and maximal expiratory mouth pressure in humans. Am. J. Respir. Crit. Care Med., v. 168, n. 6, p. 714-717, 2003.

McCONNELL, A. K.; COPESTAKE, A. J. Maximum static respiratory pressures in healthy elderly men and women: issues of reproducibility and interpretation. Respiration, v. 66, n. 3, p. 251-258, 1999.

MIZUNO, M. Human respiratory muscles: fibre morphology and capillary supply. Eur. Respir. J., v. 4, n. 5, p. 587-601, 1991.

NEDER, J. A. et al. Reference values for lung function tests. II. Maximal respiratory pressures and voluntary ventilation. Braz. J. Med. Biol. Res., Ribeirão Preto, v. 32, n. 6, p. 719-727, 1999.

OMS. Organização Mundial De Saúde. Obesity: preventing and managing the global epidemic. Report of a WHO consultation. World Health Organ Tech Rep Ser, v. 894, s. n, p. 1-253, 2000.

POLKEY, M. I.; GREEN, M.; MOXHAM, J. Measurement of respiratory muscles strength. Thorax, v. 50, n. 11, p. 1131-1135, 1995.

POWERS, S. K.; CRISWELL, D. Adaptive strategies of respiratory muscles in response to endurance exercise. Med. Sci. Sports. Exerc., v. 28, n. 9, p. 1115-1122, 1996.

SHARMA, G.; GOODWIN, J. Effect of aging on respiratory system physiology and immunology. Clin. Interv. Aging, v. 1, n. 3, p. 253-260, 2006.

STEIER, J. et al. The value of multiple tests of respiratory muscle strength. Thorax, v. 62, n. 11, p. 975-980, 2007.

SUMMERHILL, E. M. Respiratory muscle strength in the physically active elderly. Lung, v. 185, n. 6, p. 315-320, 2007. 
TOLEP, K. et al. Comparison of diaphragm strength between healthy adult elderly and young men. Am. J. Respir. Crit. Care Med., v. 152 , n. 2 , p. $677-682,1995$.

TOLEP, K.; KELSEN, S. G. Effect of aging on respiratory skeletal muscles. Clin. Chest. Med., v. 14, n. 3, p. 363-378, 1993.

VINCKEN, W.; GHEZZO, H.; COSIO, M. G. Maximal static respiratory pressures in adults: normal values and their relationship to determinants of respiratory function. Bull Eur. Physiopathol Respir., v. 23, n. 5, p. 435-439, 1987.

WATSFORD, M.; MURPHY, A. The effects of respiratory-muscle training on exercise in older women. J. Aging Phys. Act., v. 16, n. 3, p. 245-260, 2008.

WATSFORD, M. L.; MURPHY, A. J.; PINE, M. J. The effects of ageing on respiratory muscle function and performance in older adults. J. Sci. Med. Sport., v. 10, n. 1, p. 36-44, 2007. 